\title{
Los moluscos y la contaminación. Una revisión
}

\author{
Mollusks and pollution. A review
}

\author{
Erick Raúl Baqueiro-Cárdenas*, Luz Borabe, Carolina G. Goldaracena-Islas y Josefina Rodríguez-Navarro \\ Centro de Investigación en Ciencia Aplicada y Tecnología Avanzada del Instituto Politécnico Nacional, Unidad Altamira, Km. 14.5 Carretera Tampico- \\ Puerto Industrial Altamira, Altamira 89610, Tamaulipas, México. \\ *Correspondencia: ebaqueiro@gmail.com
}

\begin{abstract}
Resumen. Los moluscos son un grupo megadiverso que en cuanto a número de especies sólo lo sobrepasan los insectos y los nemátodos; no así en cuanto a la diversidad de hábitos de vida y hábitats que ocupan. Las especies de moluscos se encuentran en los desiertos y en las zonas polares; en los trópicos y en las grandes profundidades oceánicas. Sin embargo, es en las lagunas litorales tropicales donde alcanzan su máxima diversidad y función; los hay desde consumidores primarios en las redes tróficas, tanto de herbívoros como de detritívoros, hasta depredadores de segundo nivel y parásitos especializados. Existen especies especializadas y especies oportunistas, lo que se manifiesta en diferentes respuestas a las modificaciones del hábitat y la contaminación. En esta contribución se analizan las respuestas que individuos o poblaciones de moluscos tienen al impacto de la contaminación, así como sus mecanismos etológicos y fisiológicos de adaptación o sobrevivencia. Tomando en consideración lo anterior, se plantea su aplicación como indicadores de contaminación, ya sea por la desaparición de especies estenobiónticas, el predominio de las euribiónticas o por su capacidad de acumular contaminantes cuando sus concentraciones no alcanzan dosis que impacten las poblaciones, bien en procesos de bioacumulación a lo largo del ciclo de vida del organismo o por biomagnificación a través de las cadenas tróficas o de los cambios fisiológicos producidos por la contaminación.
\end{abstract}

Palabras clave: gasterópodos, bivalvos, impacto, contaminación, indicadores, bioacumulación.

\begin{abstract}
Mollusks are a mega-diverse group, surpassed only by insects and nematodes in number of species, but not in diversity of life strategies and occupied habitats; they can be found from deserts to polar zones and from the highest mountain to the deepest ocean trench. But it is at tropical coastal lagoons where they find their maximum diversity and function; there are first order consumers in both herbivore and detritus food chains, to second order carnivores and specialized parasites. The presence of specialized and opportunistic species expresses in different responses to environmental changes and pollution. In this work are analyzed the responses at individual and population levels of mollusks to pollution and pollutants. Analyzing behavioral and physiologic adaptations for survival and their usefulness as pollution indicators; given the different responses at an individual or population levels. Bearing in mind the different responses at individual or population levels, our analysis includes behavioral and physiological adaptations for survival and their usefulness as pollution indicators. Such as the disappearance of stenobiotic species, allowing the more eurybiotic species to become dominant, or for their capacity to accumulate and increase pollutant concentrations, and the phenomenon of pollutant-induced changes in physiological and morphological characteristics.
\end{abstract}

Key words: gastropods, bivalves, impact, pollution, index species, bio-acumulation.

\section{Introducción}

Los moluscos son un grupo megadiverso, sólo sobrepasado en cuanto a número de especies por los insectos y los nemátodos, más no así en cuanto a la diversidad de hábitos de vida y hábitats que ocupan. Se

Recibido: 13 noviembre 2004; aceptado: 28 marzo 2007 encuentran tanto en desiertos y zonas polares, como en trópicos y grandes profundidades oceánicas. Sin embargo, es en las lagunas litorales tropicales donde alcanzan su máxima diversidad y función. Los hay desde consumidores primarios en las redes tróficas, tanto de herbívoros como de detritívoros, hasta depredadores de segundo nivel y parásitos especializados. Existen especies sumamente especializadas, así como especies oportunistas, lo que se 
manifiesta en diferentes respuestas a las modificaciones del hábitat y la contaminación.

El número estimado de especies del phylum varía según los autores. Se da una cifra cercana a 110 mil especies (Abbott y Dance, 1982); sin embargo, consultando diferentes fuentes, se aproxima más a 80 mil, distribuidas de la siguiente manera:

Gastropoda 60000 especies, 25000 marinas, bentónicas y pelágicas; 5000 de agua dulce y 30000 terrestres. De hábitos herbívoros, carnívoros y parásitos (Abbott, 1989).

Bivalvia 15000 especies, 10000 marinas y 5000 de aguas dulces. Todas de hábitos bentónicos, principalmente filtradoras, pero las hay detritívoras, simbiontes y parásitas (Bivalvia, 2004).

Scaphopoda 350 especies marinas, bentónicas.

Aplacophora 250 especies marinas, bentónicas abisales.

Polyplacophora 650 especies marinas, bentónicas. Monoplacophora 10 especies marinas, bentónicas abisales.

Cephalopoda 1000 especies, marinas, bentónicas y pelágicas.

Las respuestas a la contaminación pueden advertirse tanto en individuos como en poblaciones y pueden ser manifestación de modificaciones en comportamiento, fisiología o simple tolerancia o intolerancia a los contaminantes. Dadas las diversas respuestas tanto de individuos como de poblaciones, se han empleado diferentes grupos de moluscos como indicadores de contaminación, sea por la desaparición de especies estenobiónticas, el predominio de especies euribiónticas, o por su capacidad de acumular contaminantes, o bien, en procesos de bioacumulación a lo largo del ciclo de vida del organismo, o biomagnificación a través de las cadenas tróficas.

Respuestas de los moluscos a la contaminación.

Fuga y atracción. La movilidad de los gasterópodos les permite evadir condiciones adversas del ambiente y fuentes puntuales de contaminación (Villarreal Chávez y Lozada López, 2004), pero en ocasiones son atraídos hacia éstas, en particular cuando las cargas de materia orgánica son altas, exponiéndose así a otros contaminantes, como es el caso de las marinas, donde son atraídos por los desperdicios de alimentos que se arrojan sobre la borda de los botes o por los organismos incrustantes de las estructuras, tanto fijas como flotantes, exponiéndose así a diversos contaminantes, entre los que destaca el Tributyltin (TBT) (Horiguchi et al., 2004).

Los bivalvos han demostrado un cierto grado de evasión. Los mejillones Dreissena polymorpha y Mytilus edulis se liberan de su biso cuando se ven sometidos a agentes irritantes, permitiendo ser arrastrados por las corrientes (Gundacker, 1994; Almeida et al., 2003), al igual que algunos bivalvos de la zona litoral que aprovechan las corrientes litorales y el oleaje para reubicarse (Van Eps, 1982). Asimismo, es bien conocida su capacidad de aislarse por varias horas con el cierre hermético de las valvas, cuando las condiciones ambientales se vuelven adversas, habiéndose demostrado este comportamiento tanto ante el estímulo de factores naturales como antropogénicos (Loosanoff y Tommers, 1948; Loosanoff, 1962; Galtsoff, 1964).

Tolerancia. Klerks y Weis (1987) hicieron una revisión profunda de la persistencia de poblaciones de moluscos en aguas contaminadas. Para ello identificaron 3 estrategias: tolerancia, aclimatación y adaptación, las que pueden adquirirse por la exposición a concentraciones subletales durante etapas previas de la vida de los organismos. De acuerdo con estos autores, las poblaciones pueden haber evolucionado resistencia genética por medio de selección natural de individuos con resistencias diferenciales. Esta tolerancia se manifiesta en la composición de las comunidades en los ecosistemas contaminados. Je et al. (2004) correlacionan los cambios en la química de los sedimentos de la bahía de Vancouver, Canadá con las variaciones en las comunidades bentónicas, donde las especies dominantes son moluscos (48\%) y poliquetos $(45 \%)$.

Los niveles de tolerancia varían con el tipo de contaminante, su concentración y la presencia de otros contaminantes. Las concentraciones de los metales pesados $\mathrm{Cu}$, Zn y Ag fueron significativamente más elevadas en ostiones, mientras que en los mejillones, fue el plomo; encontrándose Zn y Ag 3 órdenes de magnitud más elevados (Franco et al., 2002). La tolerancia no sólo varía entre especies sino que se incrementa entre organismos provenientes de ambientes contaminados. Así, Macoma balthica de ambientes contaminados con cobre presentó mayor tolerancia conforme el contenido de este metal era mayor en los sedimentos de donde provenía la población (Luoma, 1984). Aparentemente existe una aclimatación no sóloparatolerarmayores concentraciones cuandohan estado expuestos, sino que también se crea una permeabilidad que restringe el ingreso, como lo demostraron Frazier y George (1983) para Ostrea edulis. Esta especie, cuando proviene de ambientes limpios, acumula $\mathrm{Cd}$ al doble de velocidad que los ejemplares provenientes de ambientes contaminados. Pero esta tolerancia afecta en una $u$ otra forma al organismo. Weis et al. (1987) demostraron que organismos resistentes a los metales son más susceptibles a otros tipos de presiones ambientales, resultado de modificaciones o debilidad fisiológicas. 
Sobrevivencia. Sorprende la tolerancia a la contaminación de algunos moluscos que se encuentran en ambientes donde otros grupos ya han desaparecido. Como ya se mencionó, junto con los anélidos, los moluscos pueden llegar a constituir el $97 \%$ de la diversidad con un predominio del $48 \%$ (Je et al., 2004). Esto podría mal interpretarse como positivo para la diversidad de los moluscos, pues los autores no definen la disminución en diversidad con el incremento de la contaminación. Los estudios de Franco et al. (2002) registran cambios en el predominio de especies entre ostiones y mejillones de 1:5 a 4:1 ostiones por mejillón. Por el contrario, otros autores registran una alarmante pérdida de biodiversidad en los cuerpos de agua dulce del sur de los Estados Unidos y norte de México.

Según Williams et al. (1993) el norte de México y sur de los Estados Unidos, presentan la más alta biodiversidad de moluscos de agua dulce en el mundo, así como el mayor porcentaje de especies en peligro de extinción, siendo el grupo más amenazado el de los mejillones de agua dulce (Nott et al., 1995; Bogan, 1993). Sin embargo, Gillies et al. (2002) registran tasas de extinción para gasterópodos mayores a las de los bivalvos. De las 42 especies registradas para Norteamérica se consideran extintos 2 géneros. Con una pérdida del $54 \%$ de las especies en 4 años en algunos cuerpos de agua.

El incremento de la mortalidad es particularmente marcado en formas juveniles. Cataldo et al. (2001) registran mortalidades del 40-93\%, comparado con 3.3$23 \%$ de mortalidad natural en juveniles de Corbicula fluminea en ríos contaminados de Argentina, asociando la mortalidad a los niveles de contaminación en los sedimentos. La exposición a los lixiviados de las presas de jale resulta tóxico en concentraciones de sólo el 7.9\%, y en concentraciones menores causan lesiones en branquias, fusión del manto, atrofia hepática, hiperplasia y necrosis del tracto digestivo (Riba et al., 2004a, 2004b).

Comportamiento. Es bien conocida la capacidad de los bivalvos para sobrevivir en la zona intermareal, donde están sujetos a frecuentes periodos de desecación, cambios de salinidad, temperatura y fuertes cargas de sedimentos. Cuando cualquiera de estos factores se vuelve intolerante, los bivalvos lo sobrellevan cerrando sus valvas y modificando su proceso respiratorio con un incremento del ácido láctico en la hemolinfa y modificando la acidez del líquido intervalvar (Galtsoff, 1964). Un proceso fisiológico similar han demostrado Suresh y Mohandas (1987 en Lewis, 1990), como respuesta a la reducción en las tasas de filtración por exceso de cobre en el ambiente, asociado a una disminución en la tasa de captación de oxígeno. Tal tasa de filtración está asociada a una menor actividad ciliar en respuesta al incremento en los niveles de contaminantes (Abel, 1976; Almeida et al., 2003).
Las respuestas en comportamiento pueden también ser una manifestación de cambios fisiológicos inducidos por los contaminantes, ya sea en forma directa como el caso del DDT y sus derivados, que en Dreissena polymorpha induce un adelanto en el desove (Binelli et al., 2001), o indirectamente, modificando los niveles de serotonina y dopamina que tienen un efecto sobre el tono muscular y el ciclo reproductor (Almeida et al., 2003).

Bioacumulación. Diferentes especies han manifestado diferentes respuestas para cada tipo de contaminante. En términos generales, la bioacumulación y biomagnificación se dan cuando los contaminantes no interfieren con procesos vitales o las concentraciones son suficientemente bajas para permitir la tolerancia, aclimatación o adaptación del organismo.

Asimismo, la bioacumulación depende de la biodisponibilidad del contaminante: 1) la movilización de los metales en las aguas intersticiales y su especiación química; 2) transformación (p. ej., metilación) de metales como $\mathrm{As}, \mathrm{Hg}, \mathrm{Pb}$ y $\mathrm{Sn}$; 3) control ejercido por los componentes de los sedimentos (p. ej., óxido de hierro y materia orgánica); 4) competencia entre metales, como $\mathrm{Cu}$, $\mathrm{Ag}, \mathrm{Zn}$ y Cd por posiciones de ingreso en los organismos, $\mathrm{y}$ 5) los efectos de la bioperturbación, salinidad, coeficiente de redox y $\mathrm{pH}$.

Los metales pesados se toman o se toleran selectivamente. Baudo (en Lewis, 1990) encontró acumulación de $\mathrm{Cu}$ en ostiones, pero no en su depredador perforador el gasterópodo Urosalpinx cinerea. También existe una distribución diferencial en los tejidos; Horiguchi et al. (2004) encontraron diferentes concentraciones de TBT (tributiltin) en los tejidos de bivalvos, con biomagnificación hasta de 10000 veces los valores en el agua. En particular el DDT y sus derivados tienden a alcanzar concentraciones muy elevadas; en bivalvos comerciales, como ostiones, almejas y mejillones, Allsopp y Erry (2000) registran concentraciones de 0.39 a 42900 ppb. Por otro lado, los hidrocarburos aromáticos policíclicos (polycyclic aromatic hydrocarbons, PAHs) se acumulan en forma proporcional a sus concentraciones en los sedimentos.

Efectos en el metabolismo. Algunos contaminantes se incorporan a los ciclos metabólicos causando daños fisiológicos y morfológicos en los organismos, otros se tratan con estrategias fisiológicas modificadas. Frecuentemente hay evidencias de estrés.

Almeida et al. (2003) registran cambios en los niveles de 5HT (serotonina) y DOPA, tanto en la glándula digestiva como en el tejido muscular de Perna perna expuestos a metales. Asimismo, registran que los incrementos de DOPA en bivalvos pueden asociarse con diferentes presiones ambientales. DOPA produce relajación del músculo liso en Mytilus edulis, pero lo contrario, contracción del músculo 
aductor, en Anodonta cygnea, siendo además un precursor en la formación del periostraco, en tanto que la serotonina induce la relajación muscular en mejillones, y tiene además una función regulatoria en el ciclo reproductor (Almeida et al., 2003). De la misma manera, Suresh y Mohandas (1987 en Lewis, 1990) proponen los cambios de ácido láctico en la hemolinfa de los bivalvos como indicador de la respuesta de los organismos a presiones ambientales. La presencia de metales pesados induce el incremento de poros en la membrana subcelular del tracto digestivo, lo que se interpreta como una reacción al estrés (Knigge et al., 2002).

Los metales pesados se han asociado con una serie de modificaciones y adaptaciones fisiológicas en los moluscos, lo que les permite su tolerancia y bioacumulación selectiva, tanto entre especies como entre los diferentes tejidos. El incremento en la tolerancia a los metales pesados se asocia con la inducción de proteínas afines a los metales (Roesijadi, 1986; Viarengo et al., 1988a en Lewis, 1990), modificando la naturaleza y contenido de proteínas en los tejidos (Shapiro y Zvezdovskaya, 1986 en Lewis, 1990), conjuntamente a un incremento en metalotioneina, agente de enlace (Steinert y Pickwell, 1988 en Lewis, 1990).

Robinson et al. (en Lewis, 1990) encontraron distribución diferencial subcelular en el riñón de Mercenaria mercenaria: Fe y Zn se asocian a gránulos y otros organelos subcelulares, mientras que $\mathrm{Cd} \mathrm{y} \mathrm{Cu}$ se asocian a proteínas solubles y llegan a formar concreciones ricas en $\mathrm{Ca}, \mathrm{Mg}, \mathrm{Mn}, \mathrm{Zn}, \mathrm{Cd}, \mathrm{Cr}$ y $\mathrm{Cu}$ en los riñones de Argopecten circularis y Mercenaria mercenaria (Almeida et al., 2003). Ikuta (en Lewis, 1990) encontró niveles superiores de $\mathrm{Cu}$ en los filamentos del biso de todos los organismos con esta estructura, mientras que en Dreissena polymorpha se acumula $\mathrm{Cd}$ en los tejidos blandos y $\mathrm{Cu}$, $\mathrm{Zn}$ y $\mathrm{Pb}$ en los filamentos del biso, proponiendo el autor este medio como un mecanismo de limpieza o liberación de los contaminantes (Gundacker, 1994). Los mecanismos de protección, no sólo actúan en el nivel interno celular y subcelular; Tournie y Mednaoui (1986 en Lewis, 1990) encontraron que Hydrobia ulvae produce y libera al medio agentes quelantes que pueden regular la disponibilidad local del cobre.

Además de los efectos de estrés previamente mencionados, los contaminantes tienen una serie de efectos negativos en los organismos y sus poblaciones. Cheng (1988b en Almeida et al., 2003) relaciona el efecto del $\mathrm{Cu}$ sobre los ostiones con una reducción en la capacidad fagocítica sobre bacterias, reduciendo sus capacidades de defensa, lo que también identificó Matozzo et al. (2001) en Tapes philippinarum. Además, se asocian $\mathrm{Cu}$ y $\mathrm{Cd}$ con el deterioro de la porción lipídica de la membrana celular y la del lisosoma, trastornando la difusión activa (Vos et al., 1988 en Lewis, 1990, Matozzo et al., 2001) en tanto que Abel, (1976) y Almeida et al. (2003) los asocian con la reducción en la capacidad de filtración de Mytilus spp., lo cual da como resultado reducción en el potencial de crecimiento (Widdows et al., 1996).

Baqueiro et al. (2007) asocian la contaminación en general con los daños citológicos de la glándula digestiva en Crassostrea virginica, lo que disminuye la eficiencia de este órgano con los consecuentes resultados en todos los procesos fisiológicos en los que está involucrado. Respecto al DDT, se han registrado daños al ciclo reproductor de Dreissena polymorpha, induciendo adelantos en el desove y deformaciones en los oocitos (Binelli et al., 2001). El TBT se asocia con modificaciones en los ciclos sexuales de bivalvos, inhibiendo la maduración sexual y el crecimiento de las larvas, y sobreposición sexual, imposex, en gasterópodos, induciendo la sobreposición de caracteres sexuales masculinos, pene y vasos deferentes, en las hembras, con lo que se reduce la capacidad reproductora de los organismos y, por ende, el potencial reproductor de las poblaciones (Horiguchi et al., 2004).

Los daños y la adaptación han sido identificados también en cromosomas. Livingstone et al. (2000) identifican cambios cromosómicos asociados a la formación de enzimas que actúan en el bloqueo o movilización de los contaminantes (proteínas tipo CYP1-A y MXR), al daño en la membrana de los lisosomas y en la función de las membranas. Lavie y Nevo (1986) demostraron mortalidad selectiva en genotipos con aloenzimas únicas en organismos de Cerithium scabridum expuestos a metales pesados. Luoma (1984) registró la presencia de isoenzimas en poblaciones de mitílidos que indican diferencias entre poblaciones expuestas y no expuestas a metales pesados, mientras que González-Tizon et al. (2000) identificaron poblaciones poliploides de Mytilus trossulus asociadas a áreas marinas y zonas urbanas.

Moluscos como centinelas.

La tolerancia y adaptabilidad de los moluscos, en particular algunos bivalvos, los han situado como los organismos preferidos en el monitoreo de la presencia de contaminantes e indicadores de la calidad de los ecosistemas. Los bivalvos como filtradores y algunos gasterópodos como herbívoros responden, cada uno, a una fracción particular del cuerpo de agua (Conti y Cecchetti, 2003).

Como medio para identificar las fuentes de contaminación en los estuarios de Australia, Lincon-Smith y Cooper (2002) emplearon las diferentes concentraciones de metales pesados en ostiones. En 1985 se inició en los Estados Unidos de América el programa Mussel Watch que tenía como objetivo el monitoreo de la calidad de los 
cuerpos de agua interiores y costeros a través del estado de salud y presencia de contaminantes en diferentes bivalvos: Mytilus edulis y Crassostrea virginica en la costa del este, C. virginica en la del Golfo, C. rhizophorae en el Caribe, M. edulis y M. californianus en la costa oeste y en Alaska, y el mejillón zebra, Dreissena polymorpha, en los Grandes Lagos. Este programa se extendió a otros países del mundo con técnicas de muestreo y evaluación de poblaciones y el análisis estandarizado para la determinación de los diferentes tipos de contaminantes en tejidos orgánicos.

El proyecto original se diseñó para monitorear el estado y cambios temporales en metales y contaminantes orgánicos, incluidos hidrocarburos aromáticos policíclicos (PAH's) bifeniles policlorinados (PCB's), pesticidas y metales traza. Pero también para el monitoreo del estado de salud de las poblaciones de bivalvos (NOAA, 2004). Además, en todo el mundo se ha convertido en un riesgo para la salud el consumo de bivalvos, dada su capacidad de bioacumulación, no sólo de los contaminantes mencionados, sino también de bacterias y patógenos de origen humano, así como de ficotoxinas (Richards, 2003); lo cual ha llevado a la creación de un programa mundial de monitoreo de ficotoxinas, producto de marea roja, auspiciado por la UNESCO.

La determinación de los contaminantes en los tejidos de los moluscos es un indicador indiscutible de su presencia en el medio, en particular cuando sus concentraciones no son constantes o muy elevadas y su detección en agua o sedimentos se dificulta. Asimismo, la disminución en el potencial reproductor, estado fisiológico o índice de condición de las poblaciones son indicadores de la presencia de contaminantes. Mas se ha visto que la acumulación de los contaminantes es selectiva y por lo tanto no todo contaminante puede advertirse a través de los análisis de moluscos. Sin embargo, hay otros indicadores ambientales que pueden servir para la determinación de impacto ambiental por contaminación y diferentes actividades humanas; esto es, a través de los cambios en la estructura de una población de moluscos, ya sea por predominio o por debilitamiento debido a un desequilibrio en las capacidades de competencia en la comunidad. Lo cual puede lograrse mediante los siguientes mecanismos: 1 . Reducción del predominio de una población por a) pérdida de competencia, $b$ ) disminución de presa (Gasterópodos), $c$ ) incremento en depredación, $d$ ) incremento en parasitismo y e) disminución en la capacidad reproductora. 2. Predominio de una población por a) relajamiento de la competencia, b) relajamiento en la depredación, $c$ ) incremento en presa. Tales factores deben ser estudiados con relación al efecto de la contaminación y el comportamiento de las comunidades, ya que al tomar una población como indicador del estado de salud de un cuerpo de agua se pueden estar registrando señales erróneas de buena salud por el incremento de una especie altamente tolerante o adaptada a la contaminación y que por otro lado pudiera estar llevando a modificaciones en la estructura de las comunidades, con la consecuente pérdida de biodiversidad.

\section{Literatura citada}

Abel, P.D. 1976. Effect of some pollutants on the filtration rate of Mytilus. Marine Pollution Bulletin 7: 228-231.

Abbott, R. T. 1989. Compendium of landshells. American Malacologist, New York. 240 p.

Abbott, R. T. y S. P. Dance. 1982. Compendium of seashells. Dutton. 912 p.

Allsopp, M. y B. Erry. 2000. Pops in Latin America. A review of persistent organic pollutant levels in Latin America. Greenpeace Research Laboratories, Department of Biological Sciences, University of Exeter. www.greenpeace.org

Almeida, E. A., A. C. D. Bainy, M. H. G. Medeiros y P. Di Mascio. 2003. Effects of trace metal and exposure to air on serotonin and dopamine levels in tissues of the mussel Perna perna. Marine Pollution Bulletin 46:1485-1490.

Baqueiro, C. E., D. Aldana-Aranda, M. L. Sevilla y P. F. Rodríguez-Espinosa. 2007. Variations of gametogenic and spawning patterns of the oyster Crassostrea virginica from Pueblo Viejo Lagoon, Veracruz, Mexico. Transitional WatersBulletin.Bivalvia(on-line), AnimalDiversity Web. http://animaldiversity.ummz.umich.edu/site/accounts/ information/Bivalvia.html October 28, 2004.

Binelli, A., R. Bacchetta, G. Vailati, S. Galassi y A. Provini. 2001. DDT contamination in Lake Maggiore (N. Italy) and effects on zebra mussel spawning. Chemosphere 45:409-415

Bogan, A. E. 1993. Freshwater bivalve extinctions (Mollusca: Unionoida): a search for causes. American Zoologist 33:599-609.

Cataldo, D., J. C. Colombo, D. Boltovskoy, C. Bilos y P. Landoni. 2001. Environmental toxicity assessment in the Parana river delta (Argentina): simultaneous evaluation of selected pollutants and mortality rates of Corbicula fluminea (Bivalvia) early juveniles. Environmental Pollution 112:379-389.

Conti, M. E. y G. Cecchetti. 2003. A biomonitoring study: trace metals in algae and molluscs from Tyrrhenian coastal areas. Environmental Research 93:99-112

Franco, J., A. Borja, O. Solaun, y V. Pérez. 2002. Heavy metals in molluscs from the Basque Coast (Northern Spain): results from an 11-year monitoring program. Marine Pollution Bulletin 44:956-976. 
Frazier, J. M. y S. G. George. 1983. Cadmium kinetics in oysters- A comparative study of Crassostrea gigas and Ostrea edulis. Marine Biology 76:55-61.

Galtsoff, P. S. 1964. The American oyster Crassostrea virginica Gmelin. US Department of the Interior Fishery Bulletin 64:1-480.

Gillies, R. R., J. Brim-Box, J. Symanzik y E. J. Rodemaker. 2002. Effects of urbanization on the aquatic fauna of the Line Creek Watershed, Atlanta - A satellite perspective. International Journal of Remote Sensing 23:3145-3166.

González-Tizón, A. Martínez-Lage, J. Ausio y J. Méndez. 2000. Polyploidy in a natural population of mussel, Mytilus trossulus. Genome/Génome 43:409-411.

Gundacker, C. 1994. Tissue-specific heavy metal (Cd, Pb, $\mathrm{Cu}, \mathrm{Zn}$ ) deposition in a natural population of the Zebra mussel Dreissena polymorpha Pallas. Chemosphere 38:3339-3356.

Horiguchi, T., Z. Li, S. Uno, M. Shimizu, H. Shiriashi, M. Morita, J. A. Thompson y C. D. Levings. 2004. Contamination of organotin compounds and imposex in molluscs from Vancouver, Canada. Marine Environmental Research 57:75-88.

Je, J.G., T. Belan, C. Levings y B.J. Koo. 2004. Changes in benthic communities along a presumed pollution gradient in Vancouver Harbour. Marine Environmental Research 57:121-135.

Klerks, P. L. y J. S. Weis. 1987. Genetic adaptation to heavy metals in aquatic organisms: a review. Environmental Pollution 45:173-205.

Knigge, T., N. Mann, Z. Parveen, C. Perry, M. Gernhofer, R. Triebskorn, H. R. Kohler y M. Connock. 2002. Mannosomes: a molluscan intracellular tubular membrane system related to heavy metal stress? Comparative Biochemistry and Physiology Part C 131:259-269.

Lavie, B. y Nevo, E. 1986. The interactive effects of cadmium and mercury pollution on allozyme polymorphisms in the marine gastropod Cerithium scabridum. Marine Pollution Bulletin 17:21-3.

Lewis, A. G. 1990. The biological importance of copper. A literature review. Final report INCA project No. 223. http://scirus.landingzone.nl/other/?q= Type\%20y our\%20own\%20search\%20here

Lincon-Smith, M. P. y T. F. Cooper. 2002. Combining the use of gradients and reference areas to study bioaccumulation in wild oysters in the Hunter River estuary, New South Wales, Australia. Marine Pollution Bulletin 48:873-883.

Livingstone, D. R., J. K. Chipman, D. M. Lowe, C. Minier y R. K. Pipe. 2000. Development of biomarkers to detect the effects of organic pollution on aquatic invertebrates: recent molecular, genotoxic, cellular and immunological studies on the common mussel (Mytilus edulis L.) and other mytilids. International Journal of Environment and Pollution (IJEP) 13:156182.

Loosanoff, V. L. 1962. Effects of turbidity on some larval and adult bivalves. Proceedings of the Gulf and Caribbean Fisheries Institute 14:80-95.

Loosanoff, V. L. yF. D. Tommers. 1948. Effect of suspended silt and other substances on rate of feeding of oysters. Science 107:69-70.

Luoma, S. N. 1984. Significance of metal tolerance in invertebrates. AAAS Annual Meeting, May 1984, New York. p. 137-147

Matozzo, V., L. Ballarin, D.M. Pampanin y M.G. Marin. 2001. Effects of copper and cadmium exposure on functional responses of hemocytes in the clam, Tapes philippinarum. Marine Archives of Environmental Contamination and Toxicology 41:163-170.

NOAA. 2004. National Status and Trends Program, Mussel Watch. http://vertigo.hsrl.rutgers.edu/NST.html

Nott, M. P., E. Rogers y S. Pimm. 1995. Modern extinctions in the kilo-death range. Current Biology 5:14-17.

Riba I., M. Conradi, J. M. Forja y T. A. DelValls. 2004a. Sediment quality in the Guadalquivir estuary: lethal effects associated with the Aznalcollar mining spill. Marine Pollution Bulletin 48:144-152.

Riba, I., M. González de Canales, J. M. Forja y T. A. DelValls. 2004b. Sediment quality in the Guadalquivir estuary: sublethal effects associated with the Aznalcollar mining spill. Marine Pollution Bulletin 48:153-163.

Richards, G. P. 2003. The evolution of molluscan shellfish safety. In: Molluscan shellfish safety. Proceedings $4^{\text {th }}$ International Conference Molluscan Shellfish Safety, A. Villalba, B. Regueras, J. L. Romalde y R. Beiras (eds.) IOC, UNESCO. p. 221-256.

Roesijadi, G. 1986. Mercury-binding proteins from the marine mussel, Mytilus edulis. Environmental Health Perspectives 65:45-8.

van Eps G, E. 1982. Increase in surf clam populations after hypoxic water conditions off Little Egg inlet, New Jersey. Journal of Shellfish Research 2:59 - 64.

Villarreal Chávez G. y E. Lozada López. 2004. Algunos aspectos de la ecología de Nodilittorina peruviana (Lamark, 1822) y N. araucana (D’Orbigny, 1841) en la costa central de Chile. Resumen. VIII International Congress on Medical and Applied Malacology. Mexico, D.F. 35 p.

Weis, J. S., Renna, M., Vaidya, S. y P. Weis. 1987. Mercury tolerance in killifish: a stage specific phenomenon. In Oceanographic processes in marine pollution, 
vol. 1. Biological processes and wastes in the ocean, J. Capuzzo and D. Kester (eds.) Krieger, Malabar, Florida. 173-205 p.

Widdows, J., C. Nasci and V. U. Fossato. 1996. Effects of pollution on the scope for growth of mussels (Mytilus galloprovincialis) from the Venice lagoon, Italy. Marine Environmental Research 43:69-79.

Williams, J. D., M. Warren, K. Cummings, J. Harris y R. Neves. 1993. Conservation status of freshwater mussels of the United States and Canada. Fisheries 18:6-22. 